\title{
ERRATUM
}

Gereon Schares · Alfred Otto Heydorn · Arnd Cüppers

Heinz Mehlhorn · Lutz Geue · Martin Peters

Franz Josef Conraths

\section{In contrast to dogs, red foxes (Vulpes vulpes) did not shed Neospora caninum upon feeding of intermediate host tissues}

Published online: 27 March 2002

(C) Springer-Verlag 2002

\section{Parasitol Res (2002) 88:44-52}

The alteration of one word during editing the above mentioned article led to a wrong statement at the end of the discussion (last paragraph, first sentence). The mistake remained un-observed during proof-reading. The sentence "By bioassay, we can conclude that there were more than 3,700 and 200 oocysts of our
$N$. caninum-isolate among the Hammondia sp.-like oocysts collected during the first and the second foxfeeding experiments, respectively." has to be corrected into "By bioassay, we can exclude that there were more than 3,700 and 200 oocysts of our $N$. caninum-isolate among the Hammondia sp.-like oocysts collected during the first and the second fox-feeding experiments, respectively."

The online version of the original article can be found at http:// dx.doi.org/10.1007/s004360100496

G. Schares $(\bowtie) \cdot$ L. Geue $\cdot$ F.J. Conraths Institute for Epidemiological Diagnostics,

Federal Research Centre for Virus Diseases of Animals,

Seestrasse 55, 16868 Wusterhausen, Germany

E-mail: g.schares@wus.bfav.de

Fax: + 49-33979-80222

A.O. Heydorn

Institute for Parasitology

and Tropical Veterinary Medicine,

Freie Universität Berlin,

Königsweg 67, 14163 Berlin, Germany

A. Cüppers $\cdot$ H. Mehlhorn

Institut für Zoomorphologie,

Zellbiologie und Parasitologie,

Heinrich-Heine-Universität,

Universitätsstrasse 1, 40225 Düsseldorf, Germany

M. Peters

Institute for Pathology,

Tierärztliche Hochschule Hannover,

Bünteweg 17, 30559 Hannover, Germany 